\title{
Mixed convection of a micropolar fluid in a vertical channel with boundary conditions of third kind
}

\author{
J. C. Umavathi ${ }^{*}$ and Jaweriya Sultana \\ Department of Mathematics Gulbarga University, Gulbarga-585106. Karnataka. INDIA.
}

\begin{abstract}
The problem of heat source/sink on fully-developed mixed convection for the laminar flow of a micropolar fluid in a parallel plate vertical channel has been investigated analytically. The plates exchange heat with an external fluid. Both conditions of equal and of different reference temperatures of the external fluid are considered. The effect of important parameters, namely vortex viscosity parameter, ratio of Grashof number to Reynolds number and heat source/sink on the velocity, microrotation velocity and temperature have been discussed. It is found that the increase in the vortex viscosity parameter decreases the velocity for both assisting and opposing flow whereas it reduces the microrotation velocity for assisting flow and increases the microrotation velocity for opposing flow. The heat sink reduces the flow field at the right wall and increases at the left wall and converse result is observed for heat source for equal Biot numbers.
\end{abstract}

Keywords: mixed convection; micropolar fluid; boundary conditions of third kind

\section{Introduction}

The research area of micropolar fluids has been of great interest because the Navier-Stokes equations for Newtonian fluids can not successfully describe the characteristics of fluid with suspended particles. There exist several approaches to study the mechanics of fluids with a substructure. Ericksen (1960a, 1960b) derived field equations which account for the presence of substructures in the fluid. It has been experimentally demonstrated by Hoyt and Fabula (1964) and Vogel and Patterson (1964) that fluids containing small amount of polymeric additives display a reduction in skin friction. Eringen (1966) formulated the theory of micropolar fluids which display the effects of local rotary inertia and couple stresses. This theory can be used to explain the flow of colloidal fluids, liquid crystals, animal blood, etc. Eringen (1972) extended the micropolar fluid theory and developed the theory of thermo-micropolar fluids. Extensive reviews of the theory and applications can be found in the review articles by Ariman et al. (1973, 1974) and the recent books by Lukaszewicz (1999) and Eringen (2001).

Physically, micropolar fluids may be described as non-Newtonian fluids consisting of dumb-bell molecules or short rigid cylindrical element, polymer fluids, fluid suspension, etc. The presence of dust or smoke, particularly in a gas, may also be modeled using micropolar fluid dynamics. The theory of micropolar fluids first proposed by Eringen $(1966,1972)$ is capable of describing such fluids.

Studies of external convective flows of micropolar fluids have focused mainly on free, forced and mixed convection problems. Natural convection of an enclosed fluid is a long-standing classical subject. Applications are found in a variety of engineering problems, such as air conditioning of a room, solar energy collecting devices, material processing and passive cooling of nuclear reactors, to name a few. Studies of the flows of heat convection in micropolar fluids have focused mainly on flat (Ahmadi, 1976; Jena and Mathur, 1982; Ÿucel, 1989; and Rahman et al., 2006, 2007, 2008, 2009a, b) or regular surfaces (Balram and Sastry, 1973; Lien et al., 1986, 1990). Chamkha et al. (2002) analyzed numerical and analytical solutions of the developing laminar free convection of a micropolar fluid in a vertical parallel plate channel with asymmetric heating.

Kumar et al. (2005) examined the finite element solution of mixed convection micropolar fluid flow between two vertical plates with varying temperature. Cheng (2006) studied the fully developed natural convection heat and mass transfer of a micropolar fluid in a vertical channel with asymmetric wall temperatures and concentrations. Rahman et al. (2009c) have included the effects 
of variable electric conductivity and non-uniform heat source (or sink) on convective micropolar fluid flow along an inclined flat plate with surface heat flux. Umavathi et al. (2008) analysed the flow and heat transfer of a micropolar fluid sandwiched between viscous fluid layers. Prathap Kumar et al. (2010) have studied the problem of fully developed free convective flow of micropolar and viscous fluids in a vertical channel. Most recently, Umavathi and Prathap (2011) found the exact solutions for the mixed convection flow of micro-polar fluid in a vertical channel with symmetric and asymmetric wall conditions.

All the above studies on mixed convection pertain to only uniform wall temperatures in the absence of heat source/sink. Thus keeping in view the applications on micropolar fluids, the aim of this paper is to extend the analysis of Zanchini (1998) for micropolar fluid in the presence of heat source/sink. Both equal and different reference temperature of the extended fluid, as well as both different and equal Biot numbers are considered. In the absence of source/sink and for equal Biot numbers, the solutions obtained in this paper coincide with Cheng (2006). The problem can be extended to higher dimension such as spherical and cylindrical co-ordinates so that the problem will be closer to practical problems.

\section{Mathematical model}

Consider the steady and laminar flow of a micropolar fluid in the fully developed region of a parallel plate vertical channel. The $X$-axis lies on the axial plates of the channel, and its direction is opposite to the gravitational field. The $Y$-axis is orthogonal to the walls. The channel occupies the region of space $-L / 2 \leq Y \leq L / 2$ as shown in Fig. 1 . Thermal conductivity, the thermal diffusivity, the dynamic viscosity and the thermal expansion coefficient of the fluid will be assumed to be constant. The Boussineq approximation and the equation of state will be adopted.

$$
\rho=\rho_{0}\left(1-\beta\left(T-T_{0}\right)\right)
$$

Further it is assumed that the only non-zero component of the velocity field $U$ is the $X$-component of $U$. Thus, since $\Delta \cdot U=0$, we have

$$
\frac{\partial U}{\partial X}=0
$$

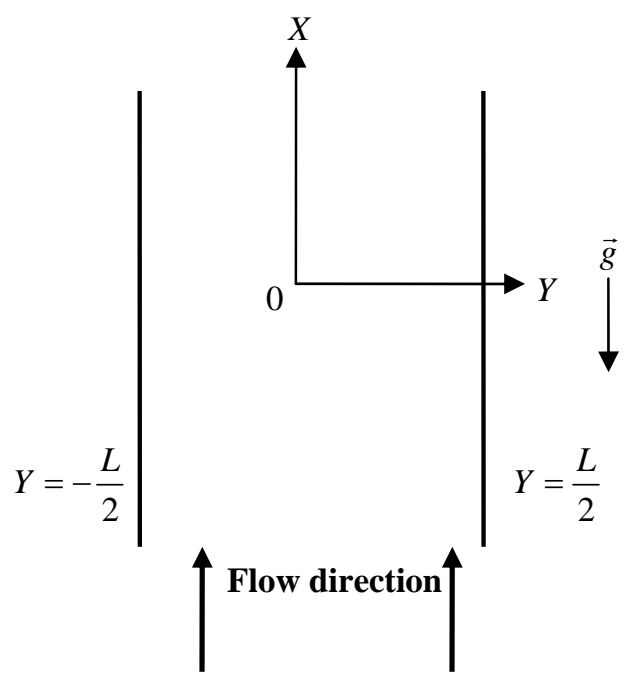

Figure 1: Physical configuration

The governing equations for micropolar fluid yield (Chamkha et al., 2002)

$$
\begin{gathered}
(\mu+\kappa) \frac{d^{2} U}{d Y^{2}}+\kappa \frac{d \bar{N}}{d Y}-\frac{\partial P}{\partial X}+\rho_{0} \beta g\left(T-T_{0}\right)=0 \\
\frac{\partial P}{\partial Y}=0 \\
\gamma \frac{d^{2} \bar{N}}{d Y^{2}}-\kappa\left(2 \bar{N}+\frac{d U}{d Y}\right)=0
\end{gathered}
$$


where $P=p+\rho_{0} g X, U$ is the velocity component in the stream wise direction, $T$ is the temperature and $\bar{N}$ is the angular velocity. Since, on account of Eq. (4), $P$ depends only on $X$, and hence Eq. (3) can be written as

$$
T-T_{0}=\frac{1}{\beta g \rho_{0}} \frac{d P}{d X}-\frac{(\mu+\kappa)}{\rho_{0} \beta g} \frac{d^{2} U}{d Y^{2}}
$$

From Eq. (6), one obtains

$$
\begin{gathered}
\frac{\partial T}{\partial X}=\frac{1}{\beta g \rho_{0}} \frac{d^{2} P}{d X^{2}} \\
\frac{\partial T}{\partial Y}=-\frac{(\mu+\kappa)}{\rho_{0} \beta g} \frac{d^{3} U}{d Y^{3}} \\
\frac{\partial^{2} T}{\partial Y^{2}}=-\frac{(\mu+\kappa)}{\rho_{0} \beta g} \frac{d^{4} U}{d Y^{4}}
\end{gathered}
$$

Both the walls of the channel will be assumed to have a negligible thickness and to exchange heat by convection with an external fluid. In particular, at $Y=-L / 2$ the external convection coefficient will be considered as uniform with the value $h_{1}$ and the fluid in the region $Y<-L / 2$ will be assumed to have a uniform reference temperature $T_{1}$. At $Y=L / 2$ the external convection coefficient will be considered as uniform with the value $h_{2}$ and the fluid in the region $Y>L / 2$ will be supposed to have a uniform reference temperature $T_{2} \geq T_{1}$. Therefore, the boundary conditions on the temperature field can be expressed as

$$
\begin{aligned}
& -\left.k \frac{\partial T}{\partial Y}\right|_{Y=-L / 2}=h_{1}\left(T_{1}-T(X,-L / 2)\right) \\
& -\left.k \frac{\partial T}{\partial Y}\right|_{Y=L / 2}=h_{2}\left(T(X, L / 2)-T_{2}\right)
\end{aligned}
$$

On account of Eq. (8), Eqs. (10) and (11) can be rewritten as

$$
\begin{aligned}
& \left.\frac{d^{3} U}{d Y^{3}}\right|_{Y=-L / 2}=\frac{\beta g h_{1}}{k(\mu+\kappa)}\left(T_{1}-T(X,-L / 2)\right) \\
& \left.\frac{d^{3} U}{d Y^{3}}\right|_{Y=L / 2}=\frac{\beta g h_{2}}{k(\mu+\kappa)}\left(T(X, L / 2)-T_{2}\right)
\end{aligned}
$$

It is easily verified that Eqs. (12) and (13) imply that $\partial T / \partial X$ is zero both at $Y=-L / 2$ and at $Y=L / 2$. Since Eq. (7) ensures that $\partial T / \partial X$ does not depend on $Y$, one is led to the conclusion that $\partial T / \partial X$ is zero everywhere. Therefore, the temperature $T$ depends only on $Y$, i.e. $T=T(Y)$. Thus, on account of Eq. (7), there exists a constant $A$ such that

$$
\frac{d P}{d X}=A
$$

For the problem under exam, the energy balance equation in the presence of heat source/sink can be written as

$$
k \frac{d^{2} T}{d Y^{2}} \pm Q\left(T-T_{0}\right)=0
$$

The boundary conditions on velocity and microrotation velocity are

$$
\begin{aligned}
& U(-L / 2)=U(L / 2)=0 \\
& \bar{N}(-L / 2)=\bar{N}(L / 2)=0
\end{aligned}
$$

Eqs. (3) to (5) (using Eq. (14)) and (15) are made dimensionless by means of the following dimensionless parameters.

$$
\begin{gathered}
u=\frac{U}{U_{0}}, \theta=\frac{T-T_{0}}{\Delta T}, y=\frac{Y}{D}, G r=\frac{g \beta \Delta T D^{3}}{v^{2}}, \operatorname{Re}=\frac{U_{0} D}{v}, \lambda=\frac{G r}{\operatorname{Re}}, R_{T}=\frac{T_{2}-T_{1}}{\Delta T}, B i_{1}=\frac{h_{1} D}{k}, \\
B i_{2}=\frac{h_{2} D}{k}, S=\frac{B i_{1} B i_{2}}{B i_{1} B i_{2}+2 B i_{1}+2 B i_{2}}, \phi=\frac{Q D^{2}}{k}, U_{0}=-\frac{A D^{2}}{48 \mu}, T_{0}=\frac{T_{1}+T_{2}}{2}+S\left(\frac{1}{B i_{1}}-\frac{1}{B i_{2}}\right)\left(T_{2}-T_{1}\right), \\
\gamma=\left(\mu+\frac{\kappa}{2}\right) D^{2}, \bar{N}=\frac{U_{0} N}{D}, K=\frac{\kappa}{\mu}
\end{gathered}
$$

In Eq. (18), $D=2 L$ is the hydraulic diameter, while the reference velocity $U_{0}$ and the reference temperature $T_{0}$ are given by

$$
U_{0}=-\frac{A D^{2}}{48 \mu}
$$




$$
T_{0}=\frac{T_{1}+T_{2}}{2}+S\left(\frac{1}{B i_{1}}-\frac{1}{B i_{2}}\right)\left(T_{2}-T_{1}\right)
$$

The reference temperature $\Delta T$ is given either by

$$
\Delta T=T_{2}-T_{1} \quad \text { if } T_{1}<T_{2} \quad \text { or } \quad \Delta T=\frac{v^{2}}{C_{p} D^{2}} \text { if } T_{1}=T_{2}
$$

On account of Eq. (14), for upward flow $A<0$, so that $U_{0}$, Re and $\lambda$ are negative. In Eq. (18), $\gamma$ is the spin gradient viscosity and we assume that $\gamma=(\mu+0.5 \kappa) j$ (Ahmadi., 1976) where $\kappa$ is the vortex viscosity and $j$ is the microinertia density. By employing the dimensionless quantities defined in Eq. (18), Eqs. (3) to (5) (using Eq. (14)) and (15) become

$$
\begin{gathered}
(1+K) \frac{d^{2} u}{d y^{2}}+K \frac{d N}{d y}+\lambda \theta+48=0 \\
\left(1+\frac{K}{2}\right) \frac{d^{2} N}{d y^{2}}-2 K N-K \frac{d u}{d y}=0 \\
\frac{d^{2} \theta}{d y^{2}} \pm \phi \theta=0
\end{gathered}
$$

The relevant boundary conditions in dimensionless form are

$$
\begin{gathered}
u(-1 / 4)=u(1 / 4)=0 \\
N(-1 / 4)=N(1 / 4)=0 \\
\left.\frac{d \theta}{d y}\right|_{-1 / 4}=B i_{1}\left(\frac{R_{T} S}{2}\left(1+\frac{4}{B i_{1}}\right)+\theta\left(-\frac{1}{4}\right)\right) \\
\left.\frac{d \theta}{d y}\right|_{1 / 4}=B i_{2}\left(\frac{R_{T} S}{2}\left(1+\frac{4}{B i_{2}}\right)-\theta\left(\frac{1}{4}\right)\right)
\end{gathered}
$$

Solving Eqs. (22) to (24) with their corresponding boundary conditions (25) to (27), gives the dimensionless temperature, velocity and microrotation velocity as

$$
\begin{gathered}
\theta=C_{1} \operatorname{Cosh}(\sqrt{\phi} y)+C_{2} \operatorname{Sinh}(\sqrt{\phi} y) \\
N=C_{3} \operatorname{Cosh}(\sqrt{\tau} y)+C_{4} \operatorname{Sinh}(\sqrt{\tau} y)+l_{5} \operatorname{Sinh}(\sqrt{\phi} y)+l_{6} \operatorname{Cosh}(\sqrt{\phi} y)+l_{7} y+l_{8} \\
u=\frac{-1}{(1+K)}\left(\frac{K}{\sqrt{\tau}}\left(C_{3} \operatorname{Sinh}(\sqrt{\tau} y)+C_{4} \operatorname{Cosh}(\sqrt{\tau} y)\right)+l_{9} \operatorname{Cosh}(\sqrt{\phi} y)+l_{10} \operatorname{Sinh}(\sqrt{\phi} y)+l_{11} y^{2}+C_{5} y+C_{6}\right)
\end{gathered}
$$

for heat absorption and

$$
\begin{gathered}
\theta=C_{1} \operatorname{Cos}(\sqrt{\phi} y)+C_{2} \operatorname{Sin}(\sqrt{\phi} y) \\
N=C_{3} \operatorname{Cosh}(\sqrt{\tau} y)+C_{4} \operatorname{Sinh}(\sqrt{\tau} y)+l_{5} \operatorname{Sin}(\sqrt{\phi} y)+l_{6} \operatorname{Cos}(\sqrt{\phi} y)+l_{7} y+l_{8} \\
u=\frac{-1}{(1+K)}\left(\frac{K}{\sqrt{\tau}}\left(C_{3} \operatorname{Sinh}(\sqrt{\tau} y)+C_{4} \operatorname{Cosh}(\sqrt{\tau} y)\right)+l_{9} \operatorname{Cos}(\sqrt{\phi} y)+l_{10} \operatorname{Sin}(\sqrt{\phi} y)+l_{11} y^{2}+C_{5} y+C_{6}\right)
\end{gathered}
$$

for heat generation respectively.

The solutions of velocity and temperature for Newtonian fluid ( $K=0$ ) with heat sink are

$$
\begin{gathered}
u=-\frac{\lambda C_{1}}{\phi} \operatorname{Cosh}(\sqrt{\phi} y)-\frac{\lambda C_{2}}{\phi} \operatorname{Sinh}(\sqrt{\phi} y)-24 y^{2}-E_{1} y-E_{2} \\
\theta=C_{1} \operatorname{Cosh}(\sqrt{\phi} y)+C_{2} \operatorname{Sinh}(\sqrt{\phi} y)
\end{gathered}
$$

The solutions of velocity and temperature for Newtonian fluid ( $K=0$ ) with heat source are

$$
\begin{gathered}
u=\frac{\lambda C_{1}}{\phi} \operatorname{Cos}(\sqrt{\phi} y)+\frac{\lambda C_{2}}{\phi} \operatorname{Sin}(\sqrt{\phi} y)-24 y^{2}-E_{3} y-E_{4} \\
\theta=C_{1} \operatorname{Cos}(\sqrt{\phi} y)+C_{2} \operatorname{Sin}(\sqrt{\phi} y)
\end{gathered}
$$

All the constants appeared in the above solutions are listed in the appendix section. 


\section{Results and discussion}

The problem of mixed convective flow and heat transfer of micropolar fluid in a vertical channel is solved analytically using boundary conditions of third kind. Two cases are considered depending on the thermal characteristics of the problem such as heat absorption and heat generation. The results are depicted graphically in Figs. 2 to 5 for heat absorption case and in Figs. 6 to 8 for heat generation case.

The effect of the mixed convective parameter $\lambda$ (ratio of Grashof number to Reynolds number) and vortex viscosity parameter $K$ on the velocity and microrotation velocity are displayed in Figs. 2a and $2 \mathrm{~b}$ respectively. As the vortex viscosity parameter $K$ increases, velocity decreases for upward $(\lambda>0)$ and downward $(\lambda<0)$ flows. It is observed from Fig. 2a that flow reversal occurs at the left wall for buoyancy assisted flow $(\lambda>0)$ and at the right wall for buoyancy opposed flow $(\lambda<0)$ with equal Biot numbers. The microrotation velocity tends to increase as the vortex viscosity parameter increases for both upward and downward flow. There is a reversal flow for microrotation velocity near left wall for buoyancy opposing flow and at the right wall for buoyancy assisting flow which is in contradiction with velocity field. The effect of $K$ on velocity and microrotation velocity is the similar results obtained in Cheng (2006).

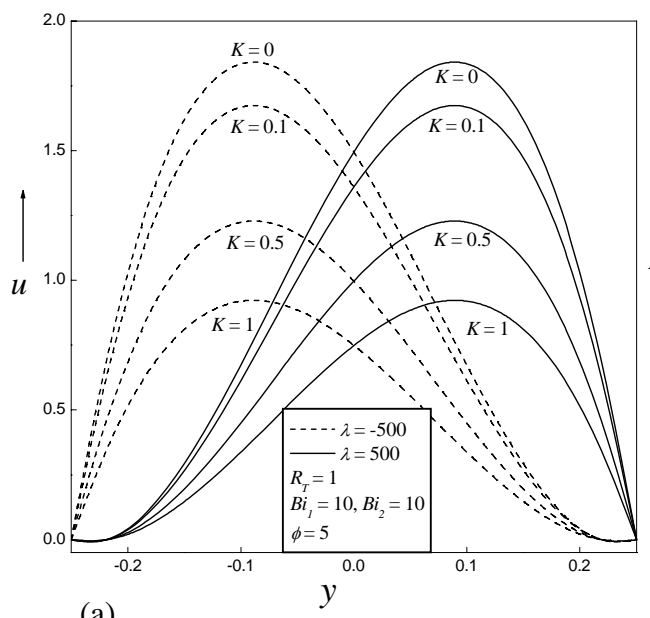

(a)

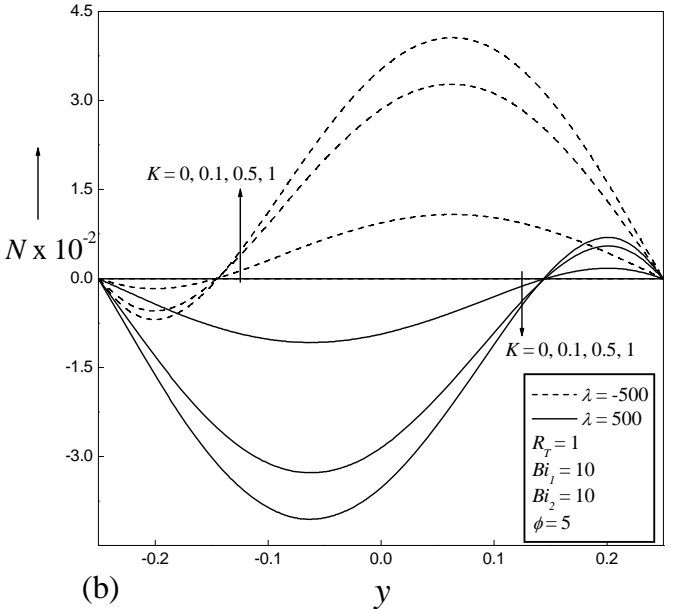

(b)

Figure 2: Plots of (a) velocity and (b) microrotation velocity for different value of $\lambda$ and $K$.
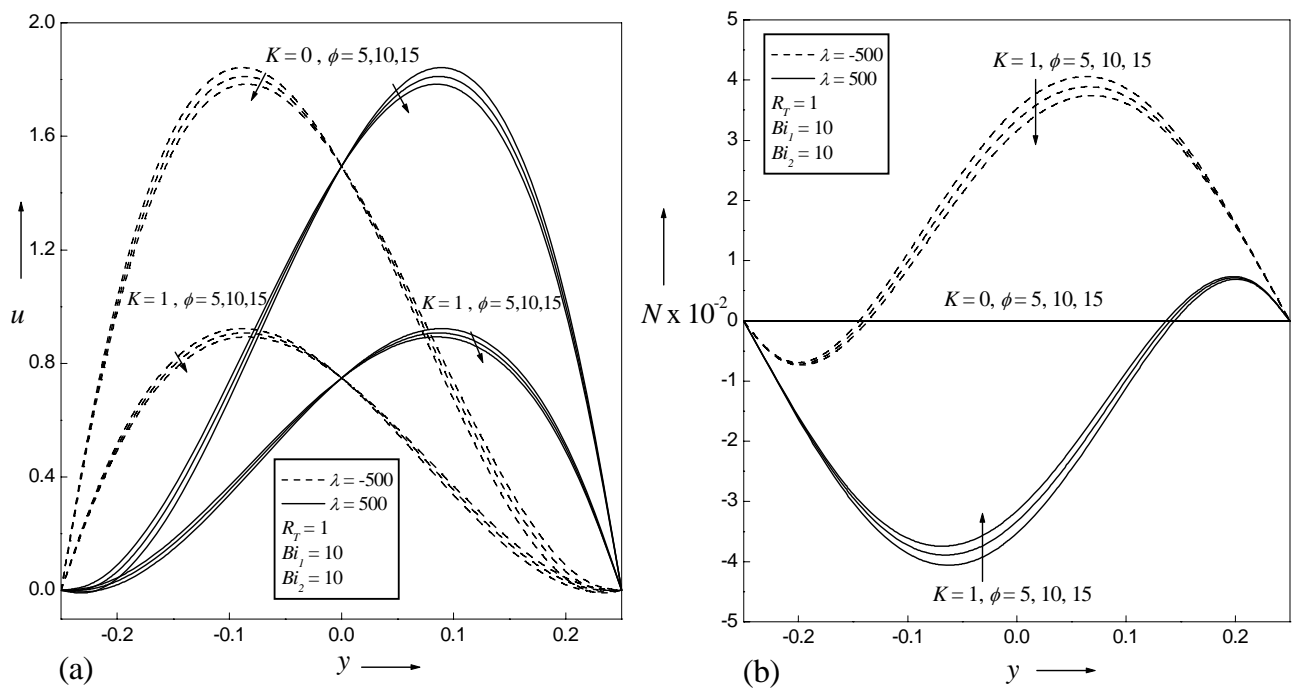

Figure 3: Plots of (a) velocity and (b) microrotation velocity for different value of $\lambda$ and $K$. 


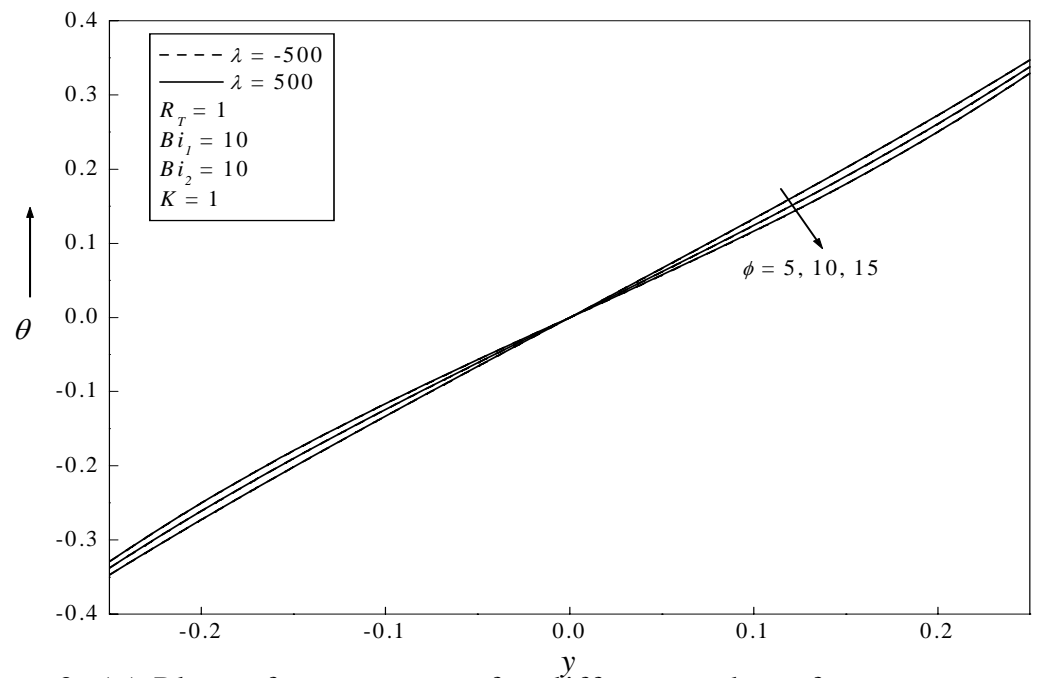

Figure 3: (c) Plots of temperature for different value of $\lambda$.

The effect of heat absorption coefficient $\phi$ on the velocity, microrotation velocity and temperature is seen in Figs. 3a, 3b and 3c respectively. It is observed that velocity decreases as heat absorption coefficient $\phi$ increases near the right wall and increases at the left wall for upward flow, whereas it increases at the right wall and decreases at the left wall for downward flow for both Newtonian $(K=0)$ and micropolar fluid $(K=1)$. As $\phi$ increases microrotation velocity decreases for buoyancy opposing flow whereas it increases for buoyancy assisting flow. It is also seen in Fig. 3b that there is no effect of $\phi$ on microrotation velocity for Newtonian fluid. As $\phi$ increases temperature decreases at the right wall and increases at the left wall for both upward and downward flow. The effect of $\phi$ on velocity and temperature for Newtonian fluid is the similar result observed in Umavathi et al. (2006).
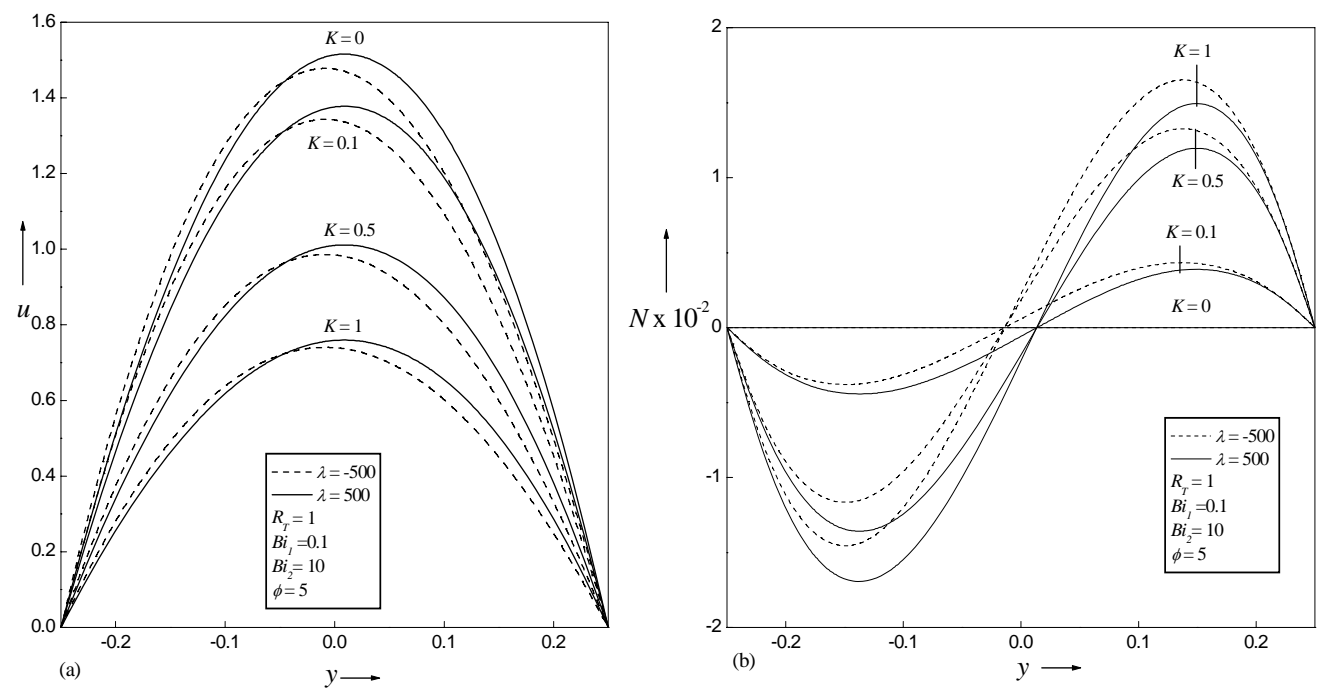

Figure 4: Plots of (a) velocity and (b) microrotation velocity for different value of $\lambda$ and $K$.

Figures $4 \mathrm{a}$ and $4 \mathrm{~b}$ shows the effect of mixed convective parameter $\lambda$ and vortex viscosity parameter $K$ on the velocity and microrotation velocity for unequal Biot numbers. As the vortex viscosity parameter $K$ increases velocity decreases for both upward and downward flow but there is no flow reversal as observed for equal Biot number (Fig. 2a). The microrotation velocity is enhanced as the vortex viscosity parameter increases for both upward and downward flow for unequal Biot numbers (Fig. 4b).

Figures 2 to 4 are the results obtained for asymmetric wall temperatures $\left(R_{T}=1\right)$. 

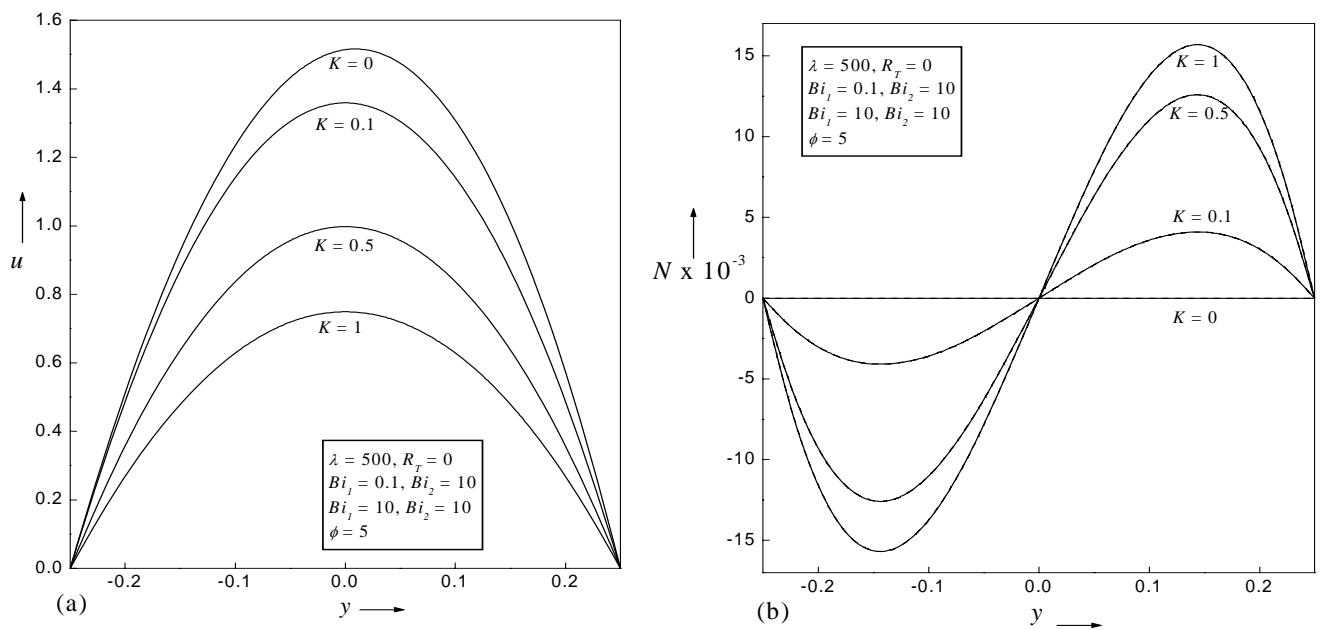

Figure 5: Plots of (a) velocity and (b) microrotation velocity for different value of $K$.

Figures 5a, b shows that velocity decreases as the vortex viscosity parameter decreases for upward and downward flow and for equal and unequal Biot numbers with symmetric wall temperatures $\left(R_{T}=0\right)$. The microrotation velocity is promoted at the right wall and suppress at the left wall as the vortex viscosity parameter $K$ increases for upward and downward flow for equal and unequal Biot numbers as seen in Fig. $5 \mathrm{~b}$ for $R_{T}=0$.
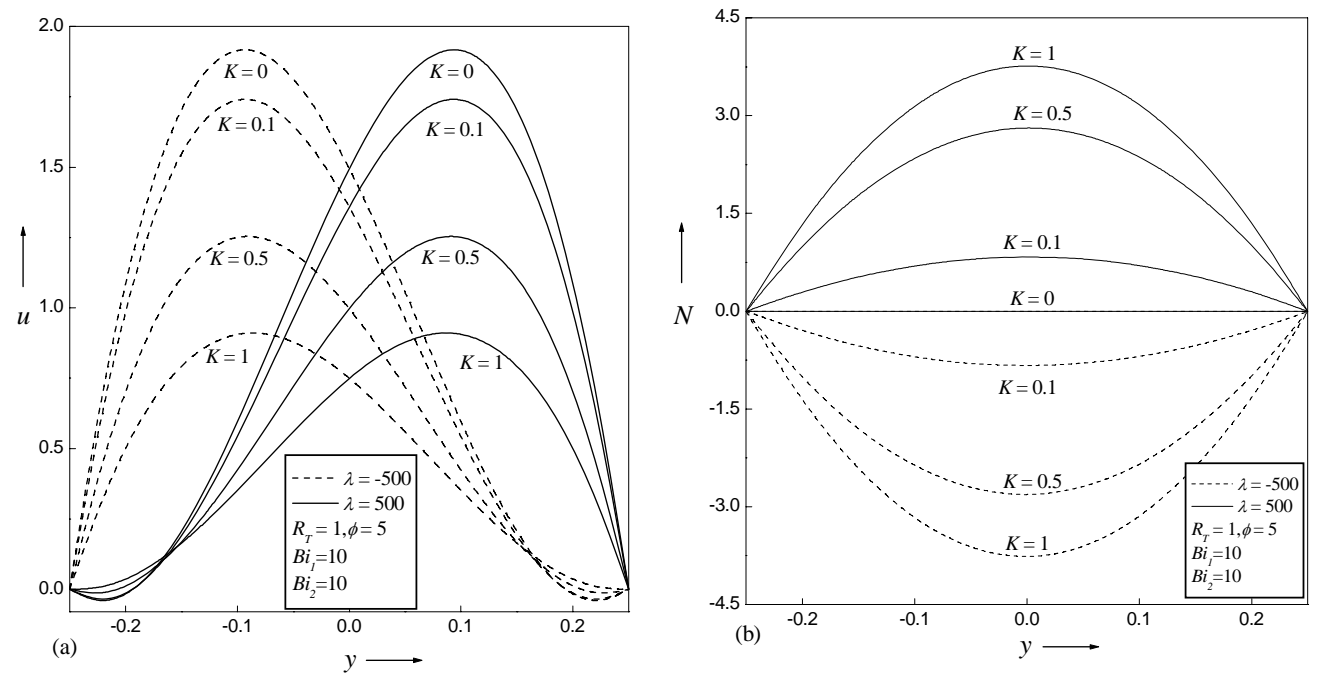

Figure 6: Plots of (a) velocity and (b) microrotation velocity for different value of $\lambda$ and K.

Figures 6 to 7 display the effect of mixed convective parameter, vortex viscosity parameter and heat generation coefficient on the velocity, microrotation velocity and temperature. The effect of mixed convective parameter $\lambda$ and vortex viscosity parameter $K$ on velocity is similar to heat absorption case (Fig. 2a). That is, for upward flow, flow reversal occurs at the left wall and for downward flow, flow reversal occurs at the right wall. For both upward and downward flows, velocity deceases as the vortex viscosity parameter increases (Fig. 6a). As the vortex viscosity $K$ increases, microrotation velocity increases for upward flow and decreases for downward flow for heat generation coefficient (Fig. 6b) which is in contradiction for heat absorption coefficient (Fig. 2b). 

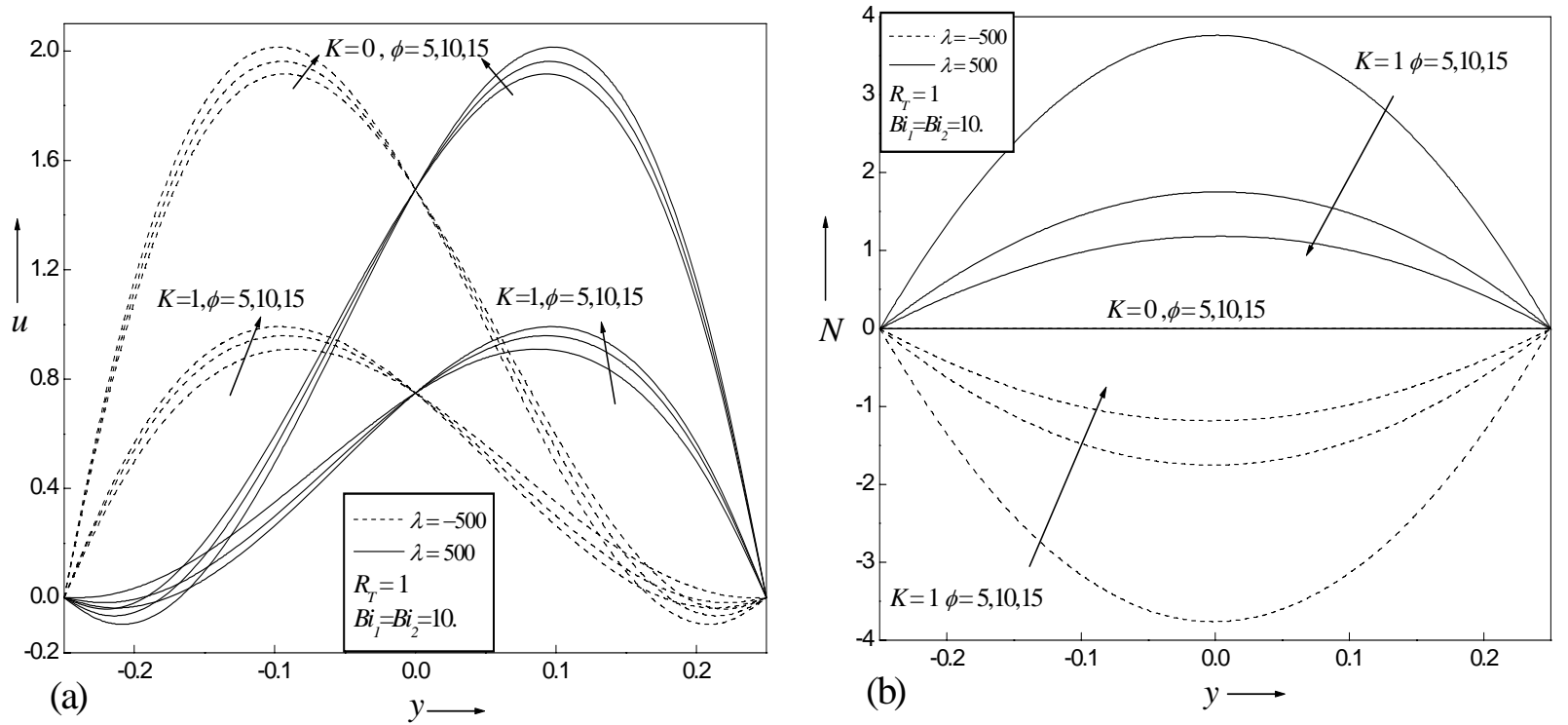

Figure 7: Plots of (a) velocity and (b) microrotation velocity for different value of $\lambda$ and $K$.

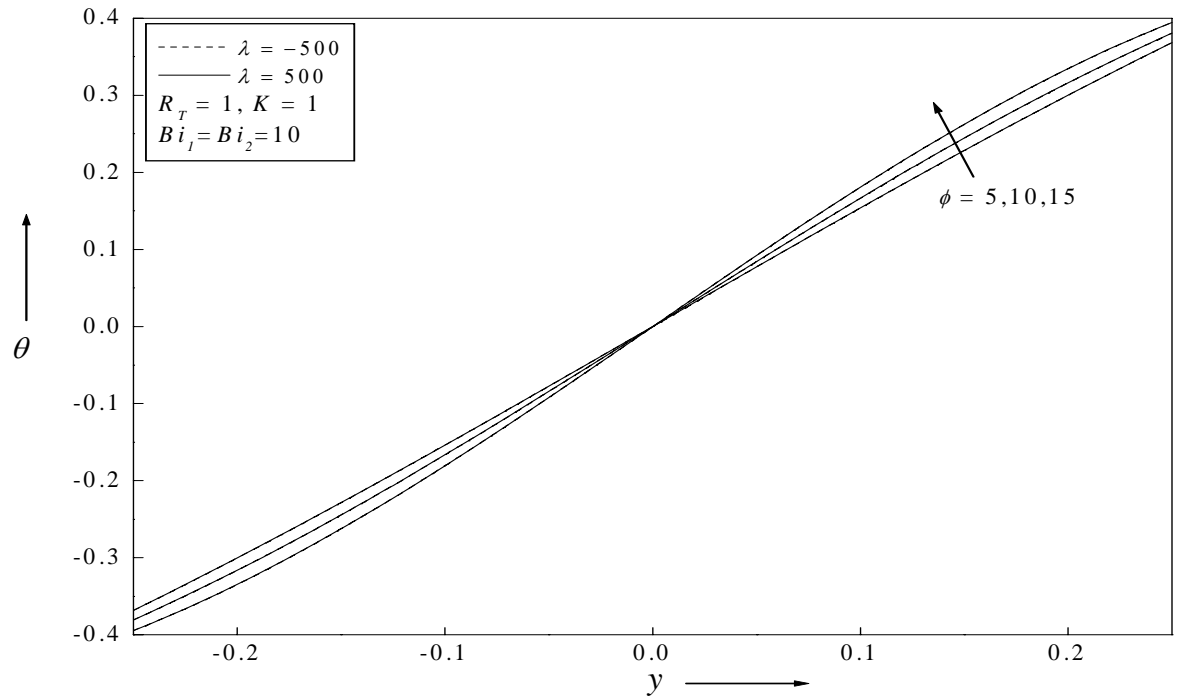

Figure 7: (c) Plots of temperature for different values of $\lambda$

Effect of heat generation coefficient $\phi$ increases the velocity at the right wall and decreases at the left wall for upward flow, whereas velocity increases at the left wall and decreases at the right wall for downward flow which is the similar result observed in Umavathi et al. (2006) for Newtonian fluid. As $\phi$ increases microrotation velocity increases for downward flow and decreases for upward flow (Fig. 7b) which is again in contradiction with heat absorption case (Fig. 3b). Effect of $\phi$ is to promote the temperature at the right wall and decreases at the left wall for both upward and downward flow (Fig. 7c) for heat source which contradicts for heat sink (Fig. 4c). Figures 6 and 7 are the profiles for equal Biot numbers.

For unequal Biot numbers with heat source, as the vortex viscosity parameter $K$ increases velocity decreases for upward and downward flows (Fig. 8a) which is the same result observed for unequal Biot numbers with heat sink (Fig. 4a). For upward flow, microrotation velocity increases as the vortex viscosity increases whereas microrotation velocity decreases as $K$ increases for downward flow as seen in Fig. 8b. 

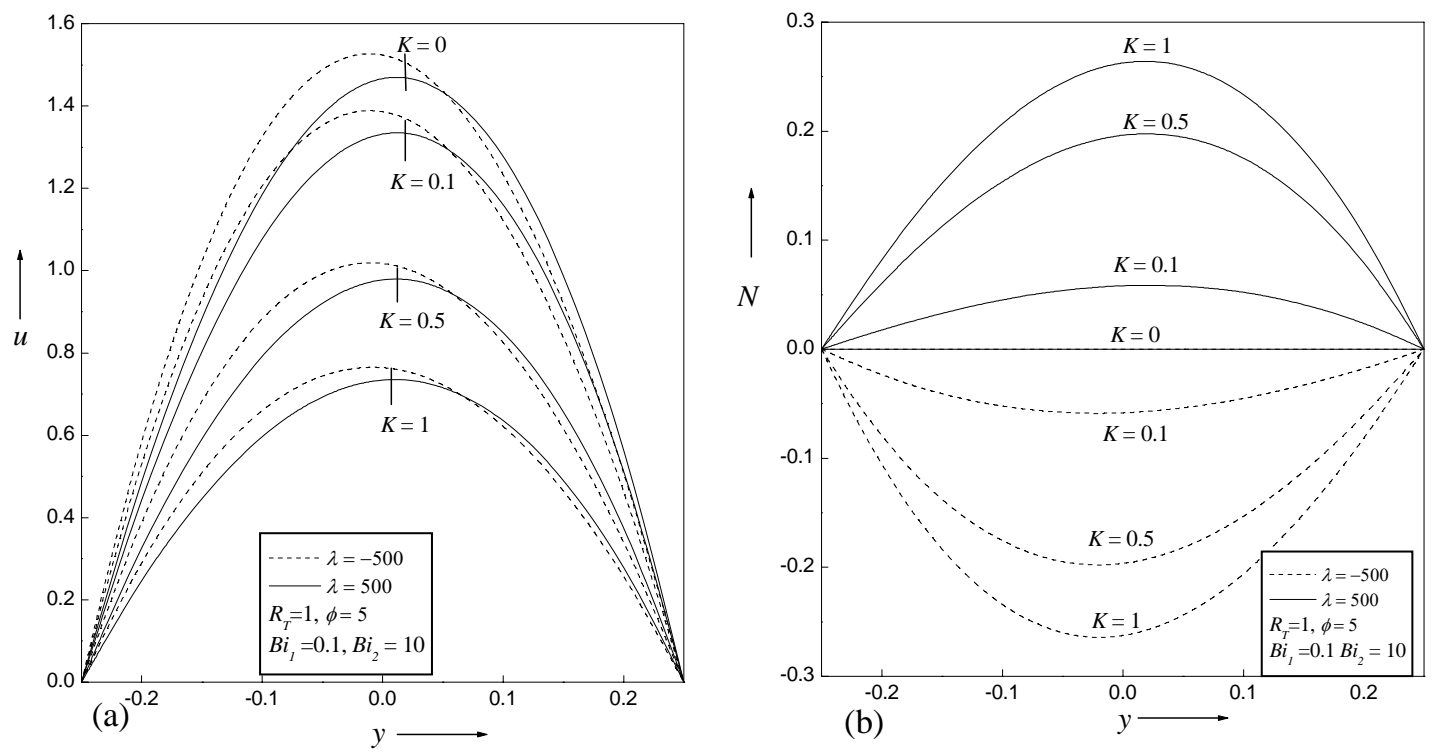

Figure 8: Plots of (a) velocity and (b) microrotation velocity for different value of $\lambda$ and $K$.

The effect of mixed convective parameter $\lambda$ and vortex viscosity parameter $K$ with symmetric wall temperatures for heat source is similar to the result observed for heat sink and hence not shown. All the above figures from 2 to 8 shows that the velocity and microrotation velocity for micropolar fluid are lower than for Newtonian fluid ( $K=0)$ which was the similar result observed by Cheng (2006).

\title{
5. Conclusions
}

The fully developed heat and mass transfer by mixed convection of a micropolar fluid in a vertical channel with heat source/sink have been studied analytically with boundary conditions of third kind. Both conditions of symmetric and asymmetric wall temperature was also analysed on the flow field. For both symmetric and asymmetric wall temperatures, the velocity decreases as the vortex viscosity parameter decreases for upward and downward flows and an increase in the vertex viscosity parameter increases the magnitude of microrotation velocity for both upward and downward flows and thus reduces the fluid flow which was the similar result obtained by Cheng (2006). The heat absorption coefficient reduces the velocity at the right wall and increases at the left wall for upward flow and contrary result is obtained for downward flow. The microrotation velocity decreased in the magnitude for upward and downward flows for heat sink. The effect of vortex viscosity parameter was to decrease the velocity and microrotation velocity for heat generation for both equal and unequal Biot numbers. The effect of heat generation coefficient was to increase the velocity at the right wall and decrease at the left wall for upward flow and for downward flow velocity increases at the left wall and decreases at the right wall. Heat generation coefficient reduces the microrotation velocity in magnitude for upward and downward flows. Heat absorption coefficient reduces the temperature at the right wall and increases at the left wall whereas a reversal effect is observed for heat generation coefficient. The results for all the parameters on the flow field for micropolar fluid were lower than those of Newtonian fluid which was the similar results obtained by Cheng (2006).

\section{Nomenclature}

\author{
$B i_{1}, B i_{2}$ Biot numbers \\ $g$ acceleration due to gravity \\ Gr Grashof number \\ $h_{1}, h_{2} \quad$ external heat transfer coefficients \\ $j \quad$ microinertia density \\ $k \quad$ thermal conductivity \\ $K \quad$ vortex viscosity parameter \\ $L \quad$ channel width \\ $\bar{N} \quad$ angular velocity of micropolar fluid
}




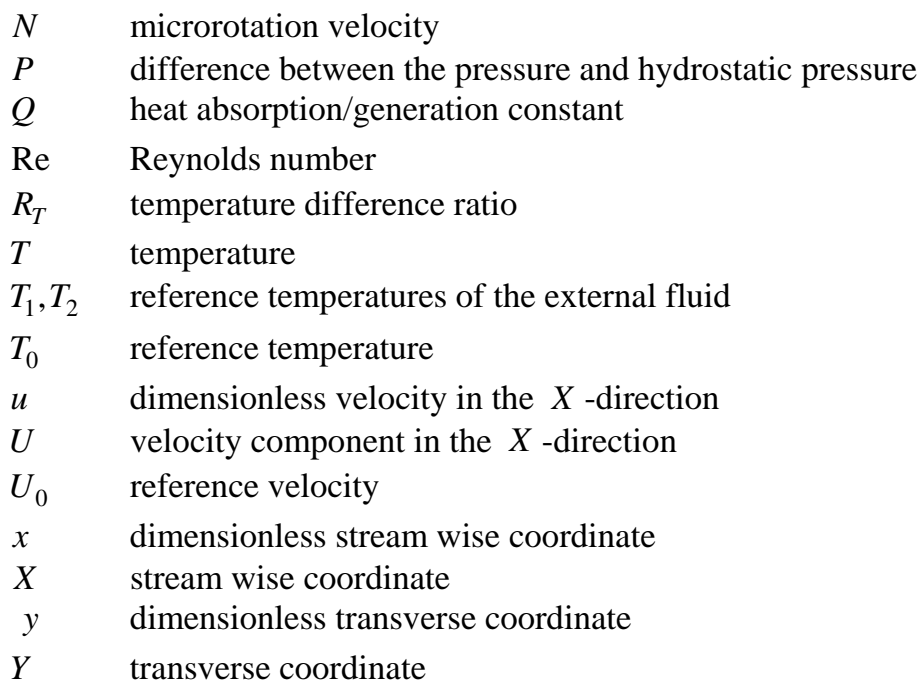

\section{Greek letters}

$\begin{array}{ll}\beta & \text { thermal expansion coefficient } \\ \phi & \text { heat absorption/generation coefficient } \\ \gamma & \text { spin gradient viscosity } \\ \kappa & \text { vortex viscosity } \\ \lambda & \text { dimensionless mixed convective parameter } \\ \mu & \text { viscosity } \\ v & \text { kinematic viscosity } \\ \theta & \text { dimensionless temperature } \\ \rho & \text { fluid density } \\ \rho_{0} & \text { value of the mass density when } T=T_{0}\end{array}$

\section{Appendix}

\section{For heat absorption}

$$
\begin{aligned}
& l_{1}=-\sqrt{\phi} \operatorname{Sinh}(\sqrt{\phi} / 4)-B i_{1} \operatorname{Cosh}(\sqrt{\phi} / 4), \quad l_{2}=\sqrt{\phi} \operatorname{Cosh}(\sqrt{\phi} / 4)+B i_{1} \operatorname{Sinh}(\sqrt{\phi} / 4), \quad b_{1}=\frac{B i_{1} R_{T} S}{2}\left(1+\frac{4}{B i_{1}}\right), \\
& l_{3}=\sqrt{\phi} \operatorname{Sinh}(\sqrt{\phi} / 4)+B i_{2} \operatorname{Cosh}(\sqrt{\phi} / 4) \quad l_{4}=\sqrt{\phi} \operatorname{Cosh}(\sqrt{\phi} / 4)+B i_{2} \operatorname{Sinh}(\sqrt{\phi} / 4), \quad b_{2}=\frac{B i_{2} R_{T} S}{2}\left(1+\frac{4}{B i_{2}}\right), \quad C_{1}=\frac{b_{1} l_{4}-b_{2} l_{2}}{l_{1} l_{4}-l_{2} l_{3}}, \\
& C_{2}=\frac{b_{1} l_{3}-b_{2} l_{1}}{l_{2} l_{3}-l_{1} l_{4}}, \quad l_{5}=-\frac{2 \tau \lambda C_{1}}{\sqrt{\phi}(2+K)(\phi-\tau)}, \quad l_{6}=-\frac{2 \tau \lambda C_{2}}{\sqrt{\phi}(2+K)(\phi-\tau)}, \quad, \\
& l_{8}=\frac{B}{(2+K)}, C_{3}=-\left(l_{6} \operatorname{Cosh}(\sqrt{\phi} / 4)+l_{8}\right) / \operatorname{Cosh}(\sqrt{\tau} / 4), C_{4}=-\left(l_{5} \operatorname{Sinh}(\sqrt{\phi} / 4)+\frac{l_{7}}{4}\right) / \operatorname{Sinh}(\sqrt{\tau} / 4) \text {, } \\
& C_{5}=4\left(\frac{-K C_{3}}{\sqrt{\tau}} \operatorname{Sinh}(\sqrt{\tau} / 4)-\left(\frac{K l_{6}}{\sqrt{\phi}}+\frac{\lambda C_{2}}{\phi}\right) \operatorname{Sinh}(\sqrt{\phi} / 4)\right), C_{6}=\frac{-K C_{4}}{\sqrt{\tau}} \operatorname{Cosh}(\sqrt{\tau} / 4)-\left(\frac{K l_{5}}{\sqrt{\phi}}+\frac{\lambda C_{1}}{\phi}\right) \operatorname{Cosh}(\sqrt{\phi} / 4)-\frac{K l_{7}}{32}-\frac{24}{16}, \\
& B=\frac{(2+K) b_{11}}{2(1+K)-(4 K / \sqrt{\tau}) T \text { an } h(\sqrt{\tau} / 4)}, b_{11}=\frac{4 K}{\sqrt{\tau}} \operatorname{Tanh}(\sqrt{\tau} / 4) l_{6} \operatorname{Cosh}(\sqrt{\phi} / 4)-\left(\frac{4 K}{\sqrt{\phi}} l_{6}+\frac{4 \lambda C_{2}}{\phi}\right) \operatorname{Sinh}(\sqrt{\phi} / 4) \text {, } \\
& l_{9}=\left(K l_{5}+\frac{\lambda C_{1}}{\sqrt{\phi}}\right) \frac{1}{\sqrt{\phi}}, \quad l_{10}=\left(K l_{6}+\frac{\lambda C_{2}}{\sqrt{\phi}}\right) \frac{1}{\sqrt{\phi}}, \quad l_{11}=\left(K l_{7}+48\right) \frac{1}{2}, \quad l_{12}=K\left(C_{1} C_{4}-C_{2} C_{3}\right) / \sqrt{\tau}(\sqrt{\tau}-\sqrt{\phi}) \text {, } \\
& l_{13}=K\left(C_{1} C_{4}+C_{2} C_{3}\right) / \sqrt{\tau}(\sqrt{\tau}+\sqrt{\phi}), l_{14}=\left(l_{9} C_{1}+l_{10} C_{2}\right) / 2 \sqrt{\phi}, l_{15}=\left(C_{2} C_{5}\right) / 2 \sqrt{\phi}-\left(l_{11} C_{1}\right) / \phi
\end{aligned}
$$


$l_{16}=2 l_{11} C_{1}(2 /(\phi \sqrt{\phi})+1 /(16 \sqrt{\phi}))-2 C_{2} C_{5} / \phi+2 C_{1} C_{6} / \sqrt{\phi}, l_{17}=\left(l_{9} C_{1}-l_{10} C_{2}\right) / 4$,

Newtonian fluid $(K=0)$

$$
\begin{aligned}
& C_{1}=\frac{b_{1} l_{4}-b_{2} l_{2}}{l_{1} l_{4}-l_{2} l_{3}}, C_{2}=\frac{b_{1} l_{3}-b_{2} l_{1}}{l_{2} l_{3}-l_{1} l_{4}} \\
& E_{1}=-\left(4 \lambda C_{2} \operatorname{Sinh}(\sqrt{\phi} / 4)\right) / \phi, E_{2}=-\left(\lambda C_{1} \operatorname{Cosh}(\sqrt{\phi} / 4)\right) / \phi-3 / 2,
\end{aligned}
$$

\section{For heat generation}

$$
\begin{aligned}
& l_{1}=-\sqrt{\phi} \operatorname{Sin}(\sqrt{\phi} / 4)+B i_{1} \operatorname{Cos}(\sqrt{\phi} / 4), \quad l_{2}=\sqrt{\phi} \operatorname{Cos}(\sqrt{\phi} / 4)+B i_{1} \operatorname{Sin}(\sqrt{\phi} / 4), \quad b_{1}=\frac{B i_{1} R_{T} S}{2}\left(1+\frac{4}{B i_{1}}\right), \\
& l_{3}=\sqrt{\phi} \operatorname{Sin}(\sqrt{\phi} / 4)+B i_{2} \operatorname{Cos}(\sqrt{\phi} / 4), \quad l_{4}=\sqrt{\phi} \operatorname{Cos}(\sqrt{\phi} / 4)+B i_{2} \operatorname{Sin}(\sqrt{\phi} / 4), \quad b_{2}=\frac{B i_{2} R_{T} S}{2}\left(1+\frac{4}{B i_{2}}\right), \quad C_{1}=\frac{b_{1} l_{4}-b_{2} l_{2}}{l_{1} l_{4}-l_{2} l_{3}}, \\
& C_{2}=\frac{b_{1} l_{3}-b_{2} l_{1}}{l_{2} l_{3}-l_{1} l_{4}}, \quad C_{3}=-\left(l_{6} \operatorname{Cos}(\sqrt{\phi} / 4)+l_{8}\right) / \operatorname{Cosh}(\sqrt{\tau} / 4) \text {, } \\
& C_{4}=-\left(l_{5} \operatorname{Sin}(\sqrt{\phi} / 4)+l_{7} / 4\right) / \operatorname{Sinh}(\sqrt{\tau} / 4), C_{5}=-4 K C_{3} \operatorname{Sinh}(\sqrt{\tau} / 4) / \sqrt{\tau}-4\left(K l_{6} / \sqrt{\phi}+\lambda C_{2} / \phi\right) \operatorname{Sin}(\sqrt{\phi} / 4) \\
& C_{6}=\frac{-K C_{4}}{\sqrt{\tau}} \operatorname{Cosh}(\sqrt{\tau} / 4)+\left(\frac{K l_{5}}{\sqrt{\phi}}+\frac{\lambda C_{1}}{\phi}\right) \operatorname{Cos}(\sqrt{\phi} / 4)-\frac{K l_{7}}{32}-\frac{24}{16}, \\
& B=\frac{(2+K) b_{11}}{2(1+K)-(4 K / \sqrt{\tau}) T \text { an } h(\sqrt{\tau} / 4)}, \\
& b_{11}=\frac{4 K}{\sqrt{\tau}} \operatorname{Tanh}(\sqrt{\tau} / 4) l_{6} \operatorname{Cos}(\sqrt{\phi} / 4)-\left(\frac{4 K}{\sqrt{\phi}} l_{6}-\frac{4 \lambda C_{2}}{\phi}\right) \operatorname{Sin}(\sqrt{\phi} / 4) \text {, } \\
& l_{5}=-\frac{2 \tau \lambda C_{1}}{\sqrt{\phi}(2+K)(\phi-\tau)}, l_{6}=\frac{2 \tau \lambda C_{2}}{\sqrt{\phi}(2+K)(\phi-\tau)}, l_{7}=\frac{48}{(2+K)}, l_{8}=\frac{B}{(2+K)} \text {, } \\
& l_{9}=-\left(K l_{5}+\frac{\lambda C_{1}}{\sqrt{\phi}}\right) \frac{1}{\sqrt{\phi}}, l_{10}=\left(K l_{6}-\frac{\lambda C_{2}}{\sqrt{\phi}}\right) \frac{1}{\sqrt{\phi}}, l_{11}=\left(K l_{7}+48\right) \frac{1}{2} \text {, } \\
& l_{12}=2 K\left(C_{1} C_{4} \sqrt{\phi}+C_{2} C_{3} \sqrt{\tau}\right) / \sqrt{\tau}(\tau+\phi), l_{13}=2 K\left(C_{1} C_{4} \sqrt{\tau}-C_{2} C_{3} \sqrt{\phi}\right) / \sqrt{\tau}(\tau+\phi), \\
& l_{14}=\left(l_{9} C_{1}-l_{10} C_{2}\right) / 2 \sqrt{\phi}, l_{15}=-\left(C_{2} C_{5}\right) / 2 \sqrt{\phi}+\left(l_{11} C_{1}\right) / \phi \text {, } \\
& l_{16}=2 l_{11} C_{1}(-2 / \phi \sqrt{\phi}+1 / 16 \sqrt{\phi})+C_{2} C_{5} / \phi-\left(2 C_{1} C_{6} / \sqrt{\phi}\right), l_{17}=\left(l_{9} C_{1}+l_{10} C_{2}\right) / 4
\end{aligned}
$$

Newtonian fluid $(K=0)$

$$
\begin{aligned}
& C_{1}=\frac{b_{1} l_{4}-b_{2} l_{2}}{l_{1} l_{4}-l_{2} l_{3}}, C_{2}=\frac{b_{1} l_{3}-b_{2} l_{1}}{l_{2} l_{3}-l_{1} l_{4}} \\
& E_{3}=\left(4 \lambda C_{2} \operatorname{Sin}(\sqrt{\phi} / 4)\right) / \phi, E_{4}=\left(\lambda C_{1} \operatorname{Cos}(\sqrt{\phi} / 4)\right) / \phi-3 / 2
\end{aligned}
$$

\section{Acknowledgement}

The authors thank UGC-New Delhi for the financial support under UGC-Major Research Project and Maulana Azad National Fellowship for Minority Students.

\section{References}

Ahmadi, G., 1976. Self-similar solution of incompressible micropolar boundary layer flow over a self-infinite plate, Int. J. Eng. Sci., Vol. 14, pp. 639-646.

Ariman, T., Turk, M.A. and Sylvester, N.D., 1973. Microcontinum fluid Mechanics - a review, Int. J. Eng. Sci., Vol. 11, pp. 905930.

Ariman, T., Turk, M.A. and Sylvester, N.D., 1974. Application of microcontinum fluid Mechanics, Int. J. Eng. Sci., Vol. 12, pp. 273-293. 
Balram, M. and Sastry, V.U.K., 1973. Micropolar free convection flow, Int. J. Heat Mass Transfer, Vol. 16, pp. 437-441.

Chamkha, A.J., Grosan, T. and Pop, I., 2002. Fully developed free convection of a micropolar fluid in a vertical channel, Int. Comm. Heat Mass Trans, Vol. 29, pp. 1021-1196.

Cheng Ching-Yang, 2006. Fully developed natural convection heat and mass transfer of a micropolar fluid in a vertical channel with asymmetric wall temperatures and concentrations, Int. Comm. Heat Mass Transfer, Vol. 33, pp. 627-635

Erickson, J.I., 1960a. Anisotropic fluids, Arch. Ration. Mech. Anal., Vol. 4, pp. 231-237.

Erickson, J.I., 1960b. Transversely isotropic fluids, Colloid Poly. Sci., Vol. 173, pp. 117-122.

Eringen, A.C., 1966. Theory of micropolar fluids, J. Math. Mech., Vol. 16, pp. 1-18.

Eringen, A.C., 1972. Theory of thermomicrofluids, J. Math. Anal. Appl., Vol. 38, pp. 480-496.

Eringen, A.C., 2001. Microcontinum field theories, II: Fluent Media, Springer, New York.

Hoyt, J.W. and Fabula, A.G., 1964. US Naval Ordinance Test Station Report.

Jena, S.K. and Mathur, M.N., 1982. Free-convection in the laminar boundary layer flow of theromocropolar fluid past a nonisothermal vertical flat plate with suction/injection, Acta Mech., Vol. 42, pp. 227-238.

Kumar, L., Bhargava, R., Bhargava, P. and Takhar, H.S., 2005. Finite element solution of mixed convection micropolar fluid flow between two vertical plates with varying temperature, Achieves of Mech., Vol. 57, pp. 251-264

Lien, F.S. and Chen, C.K., 1986. Effects of microstructure on the conjugated mixed forced and free convection-conduction analysis of heat transfer in a vertical plate fin, Trans. ASME J. Heat Trans., Vol. 108, pp. 580-584.

Lien, F.S., Chen, T.M. and Chen, C.K., 1990. Analysis of free-convection micropolar boundary layer about a horizontal permeable cylinder at a non-uniform thermal condition, Trans. ASME J. Heat Trans., Vol. 112, pp. 504-506.

Lukaszewicz, G., 1999. Micropolar fluids: Theory and application, Birkhäuser, Basel.

Prathap Kumar, J., Umavathi, J.C., Chamkha Ali, J. and Pop, I., 2010. Fully developed free convective flow of micropolar and viscous fluids in a vertical channel, App. Math. Mode., Vol. 34, pp. 1175-1186

Rahman, M.M. and Sattar, M.A., 2006. MHD convective flow of micropolar fluid past a continuously moving vertical porous plate in the presence of heat generation/absorption, ASME J. Heat Trans., Vol. 128, pp. 142-152.

Rahman, M.M. and Sattar M. A., 2007. Transient convective flow of micropolar fluid past a continuously moving vertical porous plate in the presence of radiation, Int. J. Appl. Mech. Eng., Vol. 12, pp. 497-513.

Rahman, M.M. and Sattar,M. A., 2008. Radiative heat transfer flow of micropolar fluid with variable heat flux in a porous medium, Nonlinear Anal., Model. Control, Vol. 13, pp. 71-87.

Rahman, M.M., Eltayeb, I.A. and Mujibur Rahman, S.M., 2009a. Thermo-micropolar fluid flow along a vertical permeable plate with uniform surface heat flux in the presence of heat generation, Therm. Sci., Vol. 13, pp. 23-36.

Rahman, M.M., 2009b. Convective flows of micropolar fluids from radiate isothermal porous surfaces with viscous dissipation and joule heating, Commum. Nonlinear Sci. Numer. Simulat., Vol. 14, pp. 3018-3030.

Rahman, M.M., Uddin, M.J. and Aziz, A., 2009c. Effects of variable electric conductivity and non-uniform heat source (or sink) on convective micropolar fluid flow along an inclined flat plate with surface heat flux, Int. J. Ther. Sci., Vol. 48, pp. 2331-2340

Umavathi, J.C., Prathap Kumar, J., and Chamkha, A.J., 2008. Flow and heat transfer of a micropolar fluid sandwiched between viscous fluid layers, Canadian J. Physics, Vol. 86, pp. 1-13.

Umavathi, J.C. and Prathap Kumar, J., 2011. Mixed convection flow of micropolar fluid in a vertical channel with symmetric and asymmetric wall heating conditions. Int. J. Appl. Mech. Eng., (accepted)

Vogel, W.M. and Patterson, A.M., 1964. Pacific Naval Laboratory of the Defence Research Board of Canada, Report 64-2.

Ÿucel, A., 1989. Mixed convection in micropolar fluid over a horizontal plate with surface mass transfer, Int. J. Eng. Sci., Vol. 27, pp. 1593-1608.

Zanchini, E., 1998. Effect of viscous dissipation on mixed convection in a vertical channel with boundary conditions of third kind, Int. J. Heat Mass Transfer, Vol. 41, pp. 3949-3959.

\section{Biographical notes}

J. C. Umavathi received Ph. D degree from Gulbarga University Gulbarga India in 1992. She is a Professor in the department of Mathematics, Gulbarga University, Gulbarga, Karnataka, India. Her research interest includes heat and mass transfer of multiple (Newtonian and non-Newtonian) fluids through channels and rectangular ducts, numerical simulation using Finite differences and Runge-Kutta Gill method, magnetohydradynamics, flow through porous media she has published more than 60 papers in referred International journals. She has also presented more than 20 research articles in National and International conferences. She is currently dealing with few projects sponsored by Government of India.

Jaweriya Sultana received Post Graduation in Mathematics from Gulbarga University, Gulbarga, Karnatka, India in 2007. She is working for her Doctoral degree sponsored by Maulana Azad National Fellowship for Minority Students. Her research interest includes heat and mass transfer of Newtonian and non-Newtonian fluids.

Received May 2011

Accepted June 2011

Final acceptance in revised form July 2011 